This item was submitted to Loughborough's Research Repository by the author.

Items in Figshare are protected by copyright, with all rights reserved, unless otherwise indicated.

\title{
Investigation of structural-acoustic coupling in a thin-walled reduced-scale model of a car
}

\section{PLEASE CITE THE PUBLISHED VERSION}

PUBLISHER

IOP Publishing Ltd (C) Taylor and Francis

VERSION

AM (Accepted Manuscript)

LICENCE

CC BY-NC-ND 4.0

\section{REPOSITORY RECORD}

Krylov, Victor V., Stephen J. Walsh, and Edward Winward. 2012. "Investigation of Structural-acoustic Coupling in a Thin-walled Reduced-scale Model of a Car". figshare. https://hdl.handle.net/2134/10251. 
This item was submitted to Loughborough's Institutional Repository (https://dspace.lboro.ac.uk/) by the author and is made available under the following Creative Commons Licence conditions.

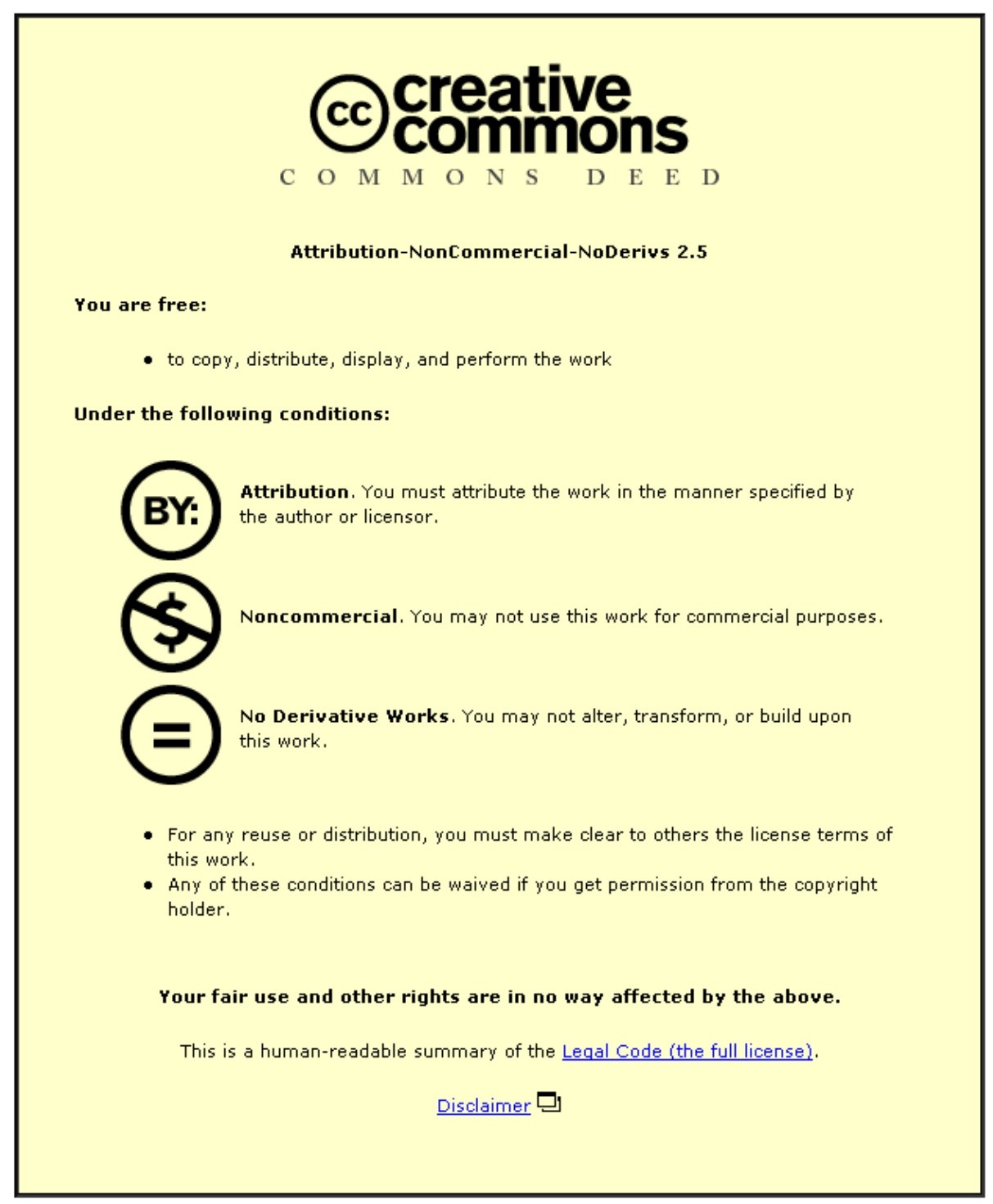

For the full text of this licence, please go to: http://creativecommons.org/licenses/by-nc-nd/2.5/ 


\title{
INVESTIGATION OF STRUCTURAL-ACOUSTIC COUPLING IN A THIN-WALLED REDUCED-SCALE MODEL OF A CAR
}

\author{
V.V. Krylov, S.J. Walsh and R.E.T.B. Winward \\ Department of Aeronautical and Automotive Engineering, Loughborough University, \\ Loughborough, Leicestershire LE11 3TU, UK
}

\begin{abstract}
The present paper describes the results of the recent research into simplified reduced-scale thin-walled models that can be used for experimental studies of vehicle interior noise. In many important cases such models can be described analytically, thus providing a developer with the effective engineering tools for prediction and mitigation of vehicle interior noise, especially on a design stage. The structural simplification in the models is based on understanding the physics of generation of predominant modes of structural vibrations by particular dynamic forces and of radiation of sound by the excited vibrations into the vehicle interior. The above-mentioned general approach is illustrated by a 1:4-scale simplified physical model of a car developed at Loughborough University - 'QUASICAR' (QUArter -Scale Interior Cavity Acoustic Rig). The model consists of a curved steel plate that is simply supported by two rigid sidewalls made of massive wooden panels. The effect of road irregularities exciting vehicle structural vibrations is imitated by electromagnetic shakers applied to the bottom of the steel plate. Measurements of structural vibrations and of the acoustic pressure generated inside the model at different positions demonstrate their good conceptual agreement with the results of theoretical predictions.
\end{abstract}

\section{KEYWORDS}

Structural-acoustic coupling, reduced-scale models, vehicle interior noise.

\section{INTRODUCTION}

Structure-borne vehicle interior noise is very complex phenomenon due to a variety of mechanisms of excitation and interaction of structural vibrations of the car body with acoustical modes of the interior cavity. A number of different modelling techniques based on finite element calculations or on combined numerical and experimental approaches have been developed, each having its own advantages and disadvantages (see, e.g. Priede (1971), Jha (1976), Nefske et al. (1982), Kim et al. (1999), Lim (2000)). Though certain important advances have been made in this field, the existing numerical or combined approaches are not accurate and robust enough to be relied upon to the extent that manufactures could dispense with the expensive process of producing prototypes for interior noise evaluation. Thus, further research is needed to improve the understanding of vehicle interior noise. 
As has been recently demonstrated by one of the present authors (Krylov (2002)), a promising and rather efficient approach to the modelling of vehicle interior noise can be based on analytical techniques employing maximum possible simplification of the model vehicle structure and of the acoustic interior. The structural simplification should be derived from understanding the physics of the problem of generation of predominant modes of structural vibrations by particular dynamic forces and of radiation of sound by the excited structural vibrations into the vehicle interior. The results of this approach can be expressed in terms of analytical formulae for sound pressure in the vehicle interior as a function of road irregularity, vehicle speed, properties of suspensions, resonance frequencies and modal shapes of structural and acoustical modes and of their coupling to each other. It can be shown that relatively few acoustic and structural modes are coupled effectively, so that only well coupled (influential) structural and acoustical modes need to be mitigated.

The present paper aims to further develop and validate this approach by carrying out experimental measurements of interior noise on a reduced-scale simplified vehicle model considered by Krylov (2002) and by comparison of the measurements' results with the theoretical predictions.

\section{THEORETICAL BACKGROUND}

It has been shown (Krylov (2002)) that, in the case of a concentrated dynamic force applied to a model vehicle body, e.g. from the front-right suspension, the structure-borne acoustic pressure in a vehicle interior can be expressed in the form

$$
p^{\prime}(\mathbf{r})=\frac{4 c^{2} \rho_{0} P_{f r}(\omega)}{\omega^{2} \rho_{0 s} h_{s} V} \sum_{m=0}^{\infty} \sum_{p=1}^{\infty} a_{m} F_{m p}(\omega) S_{m p} \Phi_{m}(\mathbf{r}) \Psi_{p}\left(\boldsymbol{\rho}_{f r}\right),
$$

where

$$
F_{m p}(\omega)=\frac{\omega^{4}}{\left(\omega_{m}{ }^{2}-\omega^{2}-2 i \delta_{m} \omega\right)\left(\omega_{p}{ }^{2}-\omega^{2}-2 i \delta_{p} \omega\right)}
$$

and

$$
S_{m p}=\frac{1}{L_{l} L_{y}} \int_{S} \Phi_{m}\left(\mathbf{r}^{\prime}\right) \Psi_{p}\left(\mathbf{\rho}^{\prime}\right) d \mathbf{r}^{\prime}
$$

The notations used in Eqns. 1-3 are the following: $P_{f r}(\omega)$ is the frequency spectrum of the applied force, $c$ and $\rho_{0}$ are sound velocity and mass density of air, $h_{s}, L_{y}$ and $\rho_{0 s}$ are thickness, width and mass density of the thin curved plate simulating an enclosed vehicle interior, $L_{l}$ is the total 'unwrapped' length of the above plate, $\Phi_{m}(\boldsymbol{r})$, and $\Psi_{p}(\boldsymbol{\rho})$ are modal shapes of acoustical and structural modes respectively, $\delta_{m}$ and $\delta_{p}$ are their attenuation decrements, and $a_{m}$ are coefficients depending on the acoustic mode type and on the shape of the enclosure. The non-dimensional function $F_{m p}(\omega)$ defined by Eqn. 2 can be termed as the frequency overlap function of the acoustical and structural modes characterised by the overall modal indexes $m$ and $p$. Similarly, the non-dimensional factor $S_{m p}$ defined by Eqn. 3 can be considered as the coefficient of structural-acoustic coupling between the corresponding acoustical and structural modes. It is the product $F_{m p}(\omega) S_{m p}$ that determines the amplitudes of the resulting acoustic pressure inside the vehicle compartment. Note that Eqns. 1-3 have been derived using the assumption of negligibly small effect of air loading on structural vibrations in a car body (for discussion of this approximation see books of Fahy (1985) and Junger \& Feit (1972). 
The Eqn. 1, in contrast to the similar expression in [6], does not consider road irregularity and the corresponding transfer function linking it with the dynamic suspension forces. Instead, for the sake of convenience of model experiments, it operates directly with the associated dynamic force $P_{f r}(\omega)$ applied from the suspension to the vehicle body structure. One can see from Eqn. 1 that the resulting acoustic pressure is formed as a summation over products of all structural and acoustic modes. However, because of the double filtration - over time and space described by the products $F_{m p}(\omega) S_{m p}$ - only relatively few of the structural and acoustical modes interact effectively and give noticeable contributions. First of all, it is clear from Eqn. 2 that only those acoustic and structural modes should be taken into account which resonance frequencies, $\omega_{m}$ and $\omega_{p}$ respectively, are close enough to each other. In addition to this, it follows from Eqn. 3 that only those overlapping acoustical and structural modes should be taken into account for which the values of the structural-acoustic coupling coefficients $S_{m p}$ are big enough.

\section{DESCRIPTION OF THE REDUCED SCALE THIN-WALLED MODEL}

To carry out experimental validation of the simplified analytical approach to prediction of vehicle interior noise described in the previous section a reduced scale model, QUASICAR, has been designed and manufactured (QUASICAR stands for QUArter Scale Interior Cavity Acoustic Rig). It is a 1/4 scale representation of an average five-door saloon. The rig consists of a curved steel plate that is simply supported by two rigid sidewalls made of massive wooden panels (Figure 1). This reflects the assumptions and outline shape of the model vehicle structure considered by Krylov (2002). The working principle of the test rig assumes that electromagnetic shakers applied to the bottom of the steel plate generate structural vibrations associated with the effect of road irregularity.

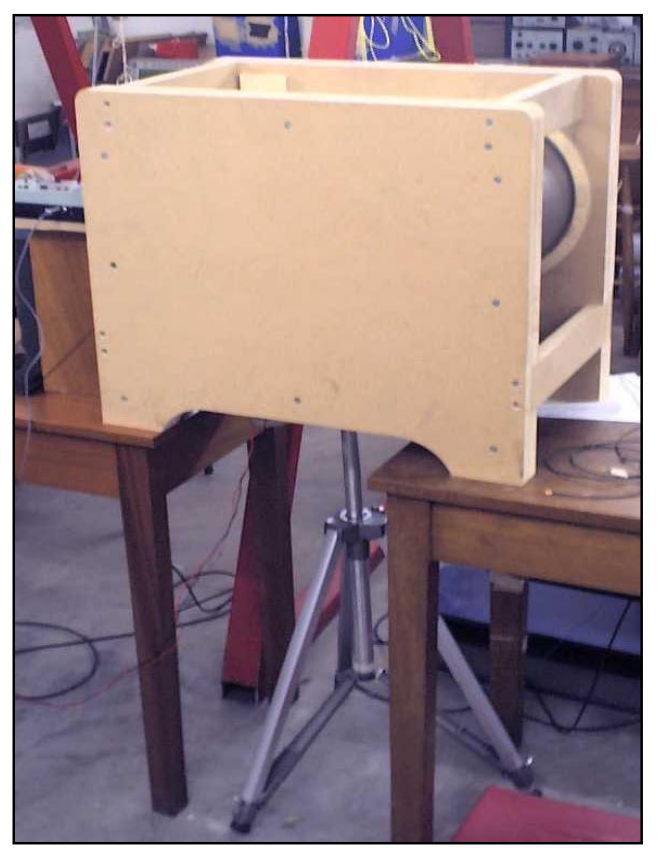

Figure 1: View of QUASICAR

The principal component in the test rig design is the curved steel plate that simulates an enclosed vehicle interior cavity. This plate is made of $20 \mathrm{SWG}$ Mild steel and is $1.2 \mathrm{~mm}$ thick. The length of the enclosed cavity is $0.5 \mathrm{~m}$, the height is $0.25 \mathrm{~m}$ and the width is $0.3 \mathrm{~m}$ (see Figure 2). The smallest radius of the plate curvature was assumed large enough in comparison with the flexural wavelengths of interest. To simulate the simply supported boundary conditions used in the theoretical model the design utilised standard circular-quadrant beading. Note that, although the curvature of the plate 
influences flexural wave dispersion characteristics, particularly at low wavenumbers, for simplicity this influence has been ignored in the theoretical model (Krylov (2002)).

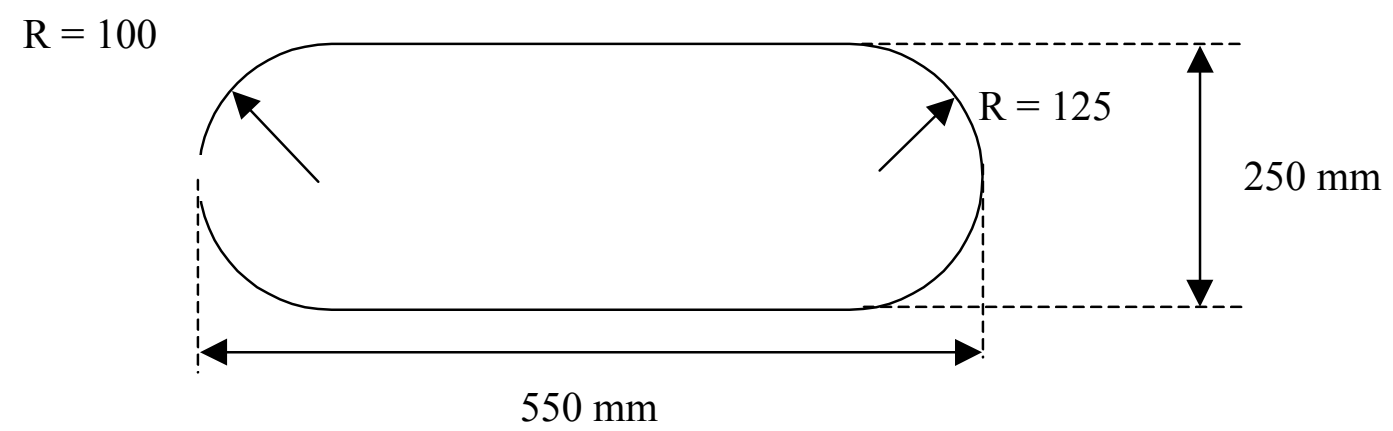

Figure 2: Vertical dimensions of the model interior cavity

The experiments were performed in the noise and vibration test facilities at the department of Aeronautical and Automotive engineering at Loughborough University. Measurements were carried out using a HP 3566 FFT analyser (see Figure 3). To measure sound pressure level (SPL), a Bruel \& Kjaer Type 2230 sound pressure level meter was used as well as microphones. A pair of Bruel \& Kjaer Type 2635 charge amplifies provided the amplification from the sensor equipment to the analyser.

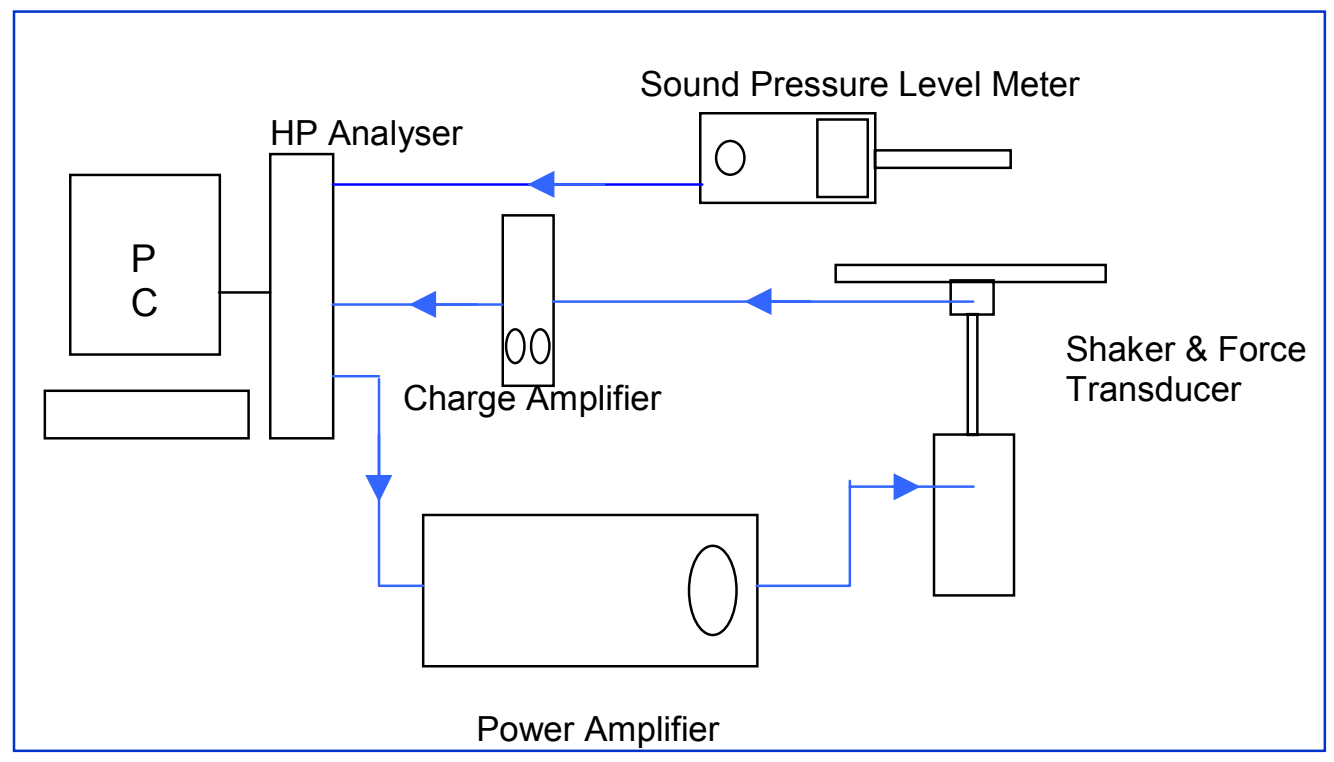

Figure 3: Measurements layout

Structural tests were conducted using a Ling Dynamic Systems 200 electromagnetic shaker and ICP type 352M24 accelerometers. The location of the shaker was associated with the position of the frontright suspension of a vehicle. The shaker was secured to a tripod and this was positioned underneath the QUASICAR rig (see Figure 1). To provide the acoustic excitation in the frequency range from 200 $-1000 \mathrm{~Hz}$ a miniature Visaton medium range loudspeaker (with a diameter of $50.5 \mathrm{~mm}$ ) was used. The excitation signal was a continuous white noise generated using the HP 3566 FFT analyser.

\section{EXPERIMENTAL RESULTS AND THEIR DISCUSSION}

The experimental programme included three main sets of experiments carried out on QUASICAR: a) acoustic excitation testing; b) structural excitation testing; and c) structural-acoustic testing. The 
objectives of these tests were respectively to determine acoustic resonance properties of the QUASICAR interior cavity, to investigate structural properties (point mobility) of the plate section of the QUASICAR, and to measure structural-acoustic response inside the cavity. Structural measurements were carried out using ICP type 352M24 accelerometers on the flat lower section of the plate at the location of the electromagnetic shaker. Acoustic measurements inside the cavity were conducted using both an SPL meter and microphones.

Figure 4 illustrates the acoustic response of QUASICAR to a loudspeaker excitation measured by an SPL meter in the frequency range $200-1000 \mathrm{~Hz}$, with the speaker being located near the corner and subject to random white noise excitation. Measurements shown on Figure 4 have been carried out for a front gap of the cavity covered with the inserted foam. The four observed resonant peaks are at 368 , 582, 683 and $886 \mathrm{~Hz}$. These observations agree well with the theoretical predictions following from the simplified theoretical model (Krylov (2002)).

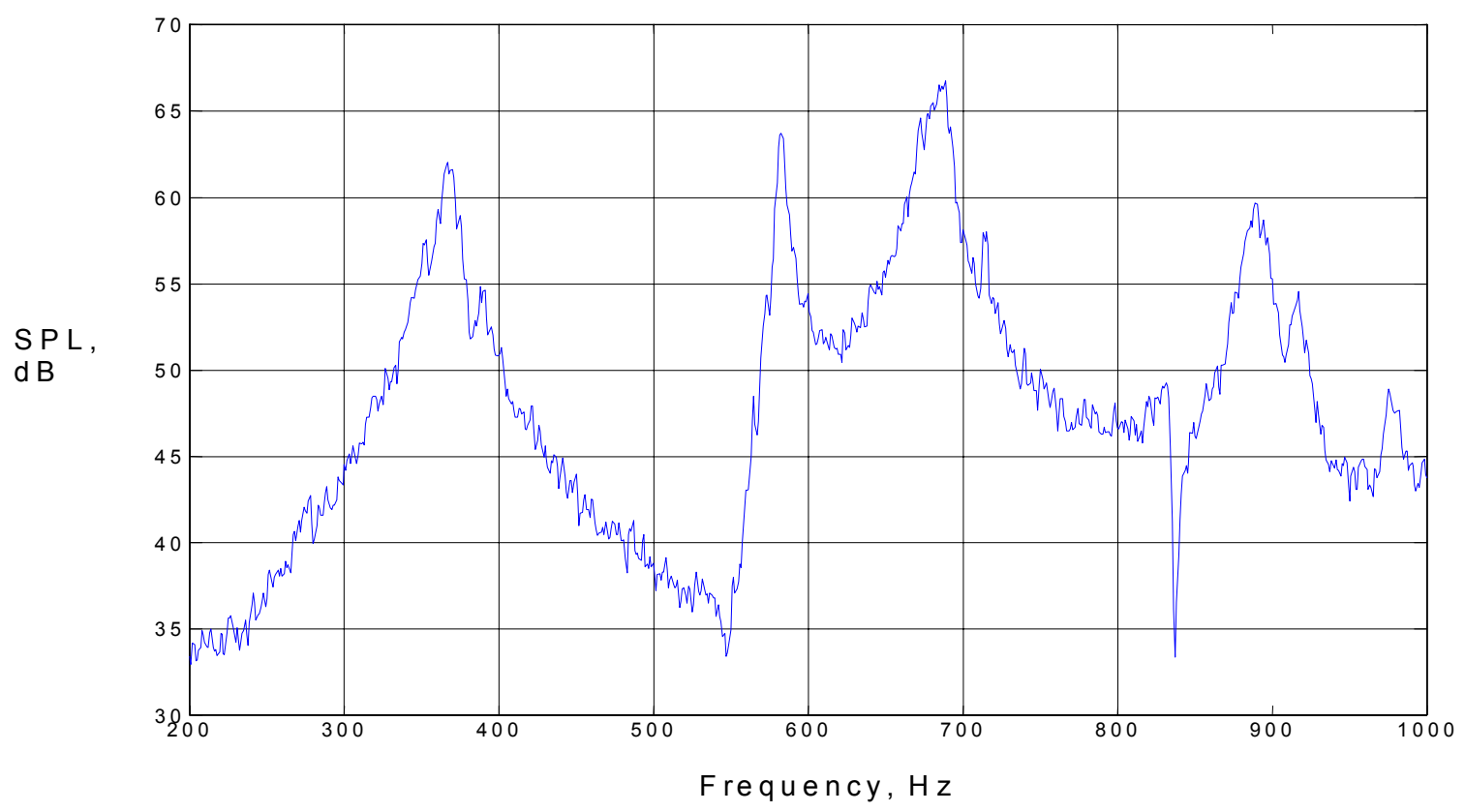

Figure 4: Acoustic response of the QUASICAR interior cavity

Figure 5 shows a normalised acoustic response of QUASICAR in a wider frequency range $(200-3500$ $\mathrm{Hz}$ ). Curve 1 describes experimental results, and curve 2 shows theoretical predictions based on the acoustic Green's function for the equivalent rectangular enclosure (Krylov (2002)). One can see that at relatively low frequencies the theoretical predictions agree well with the experiments, whereas at higher frequencies the agreement is quite poor. The latter had to be expected since at higher frequencies the difference between the actual geometry of the QUASICAR cavity and the equivalent rectangular enclosure becomes more essential.

The results for structural excitation tests in the frequency range $200-3500 \mathrm{~Hz}$ are presented in Figure 6 for a shaker located in the position of the front-right suspension. Curve 1 describes experimental results, and curve 2 shows theoretical predictions based on the structural Green's function for the approximating simply supported flat plate (Krylov (2002)). In this case, as expected, the agreement between the theory and the experiment is better at higher frequencies corresponding to shorter wavelengths of flexural waves propagating in the plate. It is well known that for short wavelengths, as compared to the minimum radius of curvature, the assumption of negligible influence of plate curvature is justified. 
Figure 7 illustrates the structural-acoustic response of QUASICAR. For convenience, a structural response is shown on Figure 7 as well. A visual comparison of the structural response, the corresponding structural-acoustic response (reading of the SPL meter) and the acoustic response (see Figure 4) shows very good conceptual agreement with the theoretical model (Krylov (2002)).

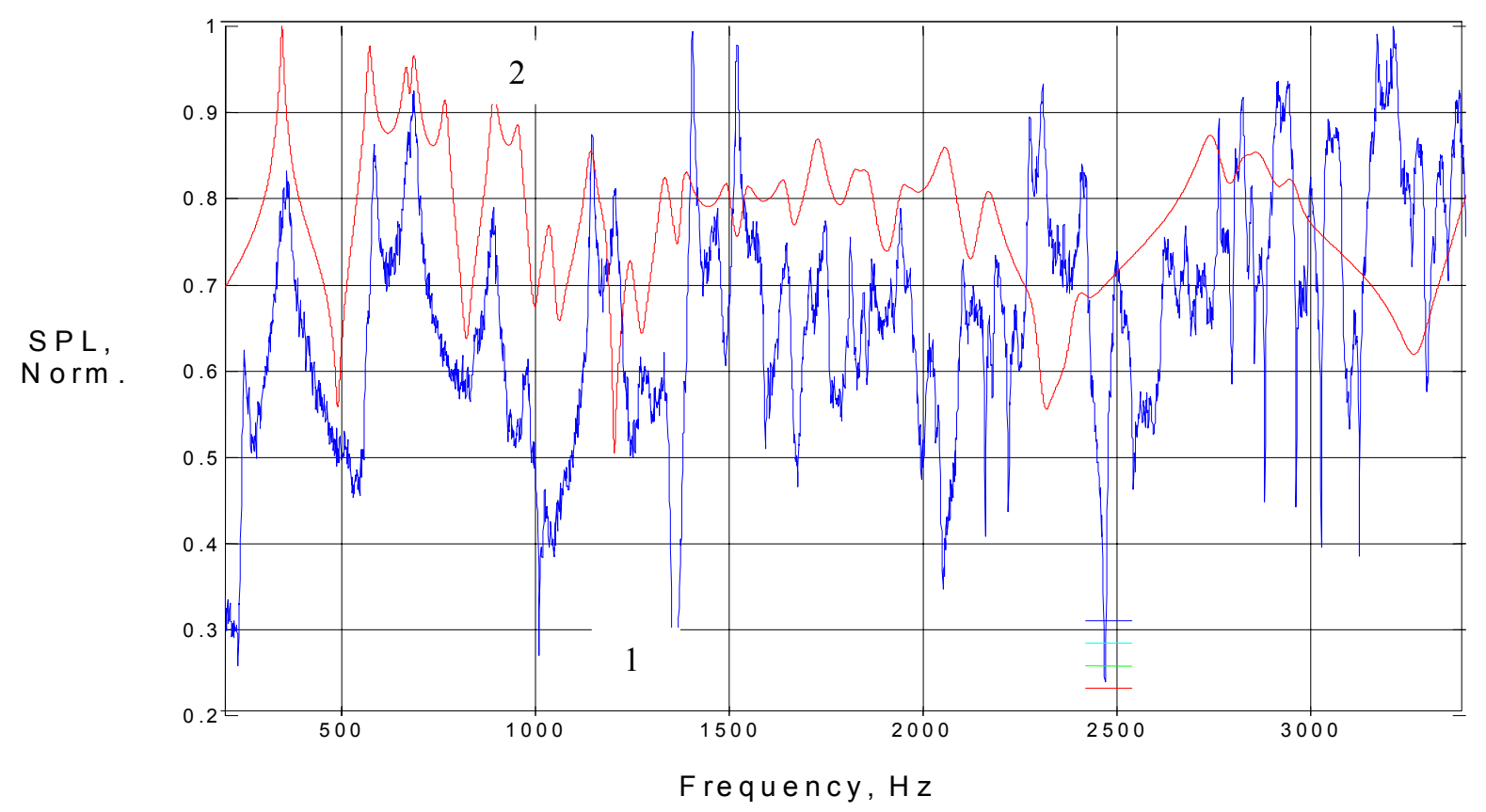

Figure 5: Normalised acoustic response of the QUASICAR interior cavity in a wider frequency range; 1 - experiment, 2 - theory

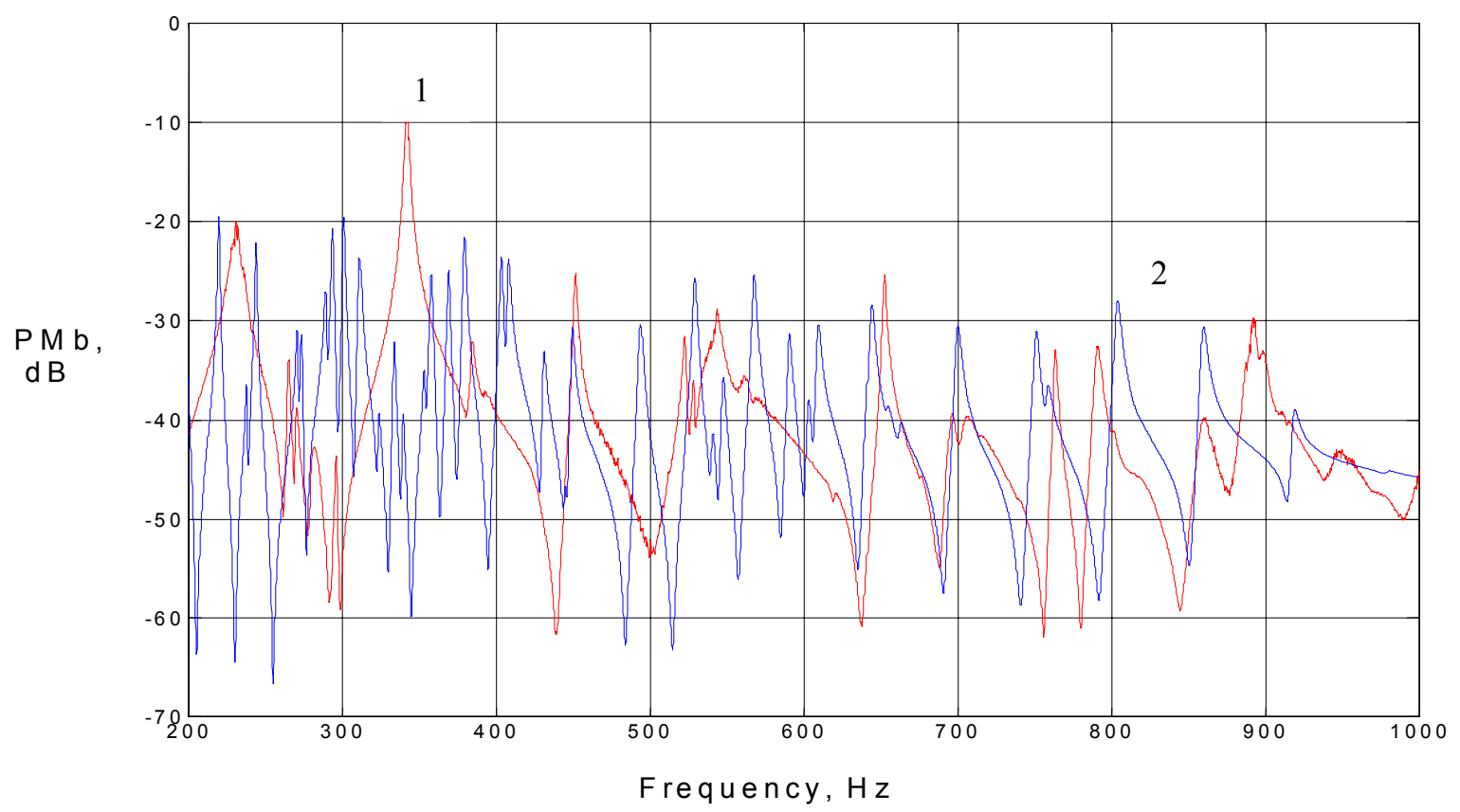

Figure 6: Structural response of the QUASICAR; 1 - experiment, 2 - theory

The degree of association between the measured point mobility and the structural-acoustic response is considerable and illustrates the importance of breaking down the analysis of interior noise in 
QUASICAR into both the structural and acoustic properties and then determining the coupling characteristics between these properties. By comparison of structural and structural-acoustic responses, one can see that the principal resonance frequencies of the structure are being 'transmitted' into the peaks of structural-acoustic response, and the resulting frequency peaks of structural-acoustic response are even more pronounced if they are close to acoustic resonant frequencies (see Figure 4).
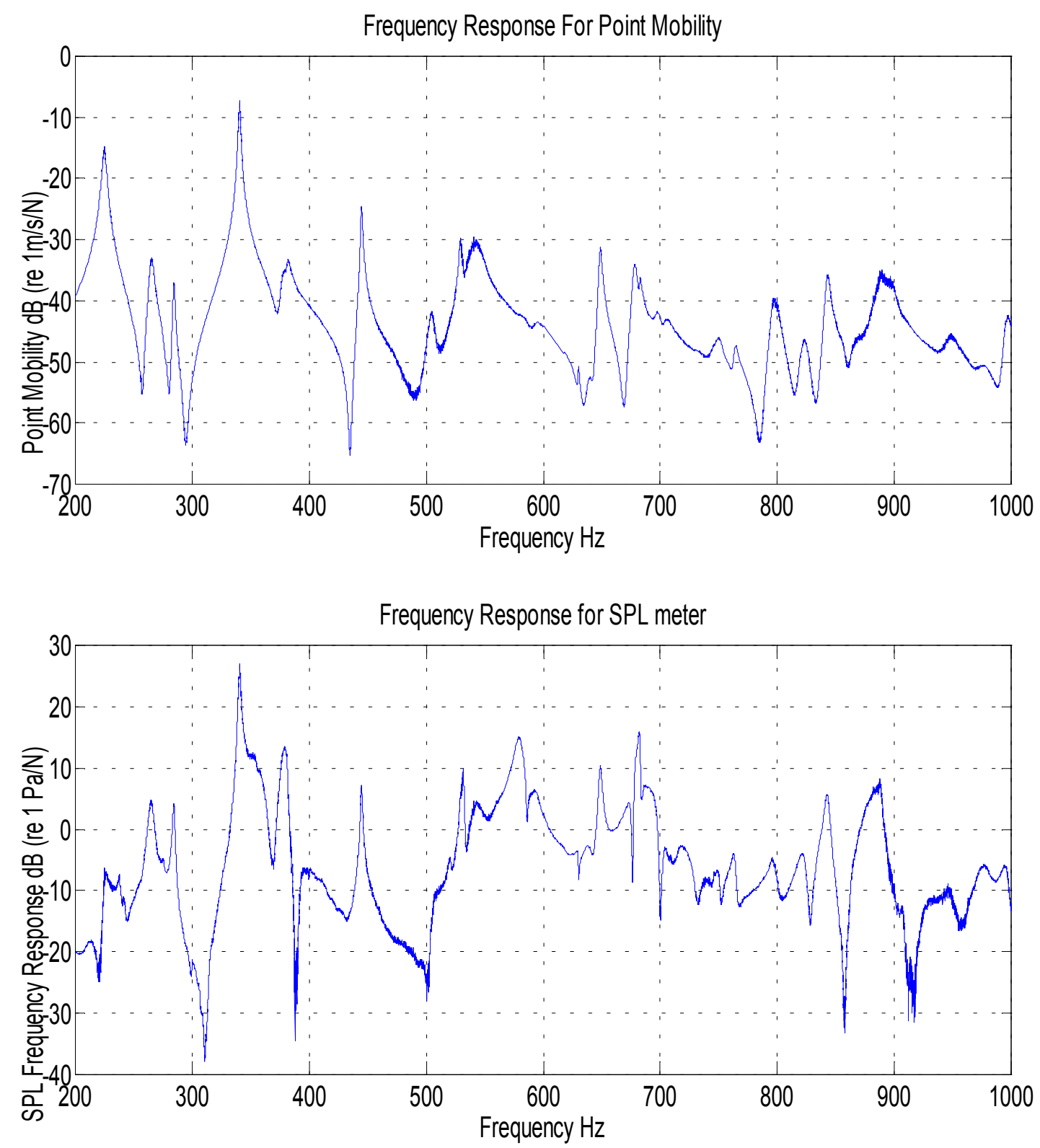

Figure 7: Structural and structural-acoustic responses of the QUASICAR

In agreement with the general concept of the model, it can be clearly seen from Figures 7 and 4 that the acoustic resonances result in considerable amplification of the structural response, leading to high in-cavity noise levels. The most important structural resonant frequencies measured in the QUASICAR are located at 342, 370, and $440 \mathrm{~Hz}$, the primary structural resonant frequency being approximately $342 \mathrm{~Hz}$. The nearest acoustic resonance frequency is $368 \mathrm{~Hz}$. The coupling of structural modes at the above-mentioned structural resonant frequencies with the acoustic mode centered at $368 \mathrm{~Hz}$ leads to a significant response in the structure-borne acoustic field at about 342, 370 , and $440 \mathrm{~Hz}$. Remembering to scale these results by $1 / 4$, this is equivalent to frequencies of 85.5 , 
92.5, and $110 \mathrm{~Hz}$ for an actual vehicle. Similarly, considering the acoustic resonances at 582, 683 and $886 \mathrm{~Hz}$ and combining them with the structural ones, one can explain the observed peaks of structuralacoustic response at higher frequencies.

Note that, whereas a conceptual agreement between the theory (Krylov (2002)) and the experiments is very good, the quantitative difference between predicted and measured values of structural-acoustic response is quite significant. This is not surprising, considering the above-mentioned facts of inadequate behaviour of the predicted acoustic response at higher frequencies (see Figure 5) and of the structural response at lower frequencies (see Figure 6). Apparently, the neglect of the plate curvature effects on structural response and using an equivalent rectangular enclose to calculate the acoustic response should be considered as oversimplification, and further improvement of the theoretical model is needed.

\section{CONCLUSIONS}

The reported work on reduced-scale vehicle models has lead to the design and development of a QUASICAR test rig and to the experimental testing and validation of the basic concepts and abilities of the simplified analytical approach to prediction of vehicle interior noise. It is intended that during the next phases of the project, this work will be further expanded to address the effects of plate curvature, attached panels and of different types of structural excitation on the behaviour of structural and acoustical modes as well as on the resulting interior noise.

Although QUASICAR represents one of the simplest designs of model experimental rig, it is expected that main results on excitation of structural vibrations and generation of interior noise obtained on this rig will be similar also for more complex rigs reflecting most important features of real vehicles. The authors believe that introduction of such more sophisticated but still manageable reduced-scale models will lead to the development of efficient analytical and experimental tools for identification of main contributors to vehicle interior noise that will assist in its more reliable prediction and mitigation.

\section{REFERENCES}

Fahy, F. (1985). Sound and Structural Vibration, Academic Press, London, UK.

Jha, S.K. (1976). Characteristics and sources of noise and vibration and their control on motor cars. Journal of Sound and Vibration 47, 543-558.

Junger, M.C. and Feit, D. (1972). Sound, Structures and their Interaction, MIT Press, Cambridge MA, USA.

Kim, S.H., Lee, J.M. and Sung, M.H. (1999). Structural-acoustic modal coupling analysis and application to noise reduction in a vehicle passenger compartment. Journal of Sound and Vibration 225, 989-999.

Krylov, V.V. (2002). Simplified analytical models for prediction of vehicle interior noise. Proceedings of the International Conference on Noise and Vibration Engineering (ISMA 2002), Leuven, Belgium, 16-18 Sept. 2002, Ed. P. Sas, Vol. V, pp. 1973-1980.

Lim, T.C. (2000). Automotive panel noise contribution modelling based on finite element and measured structural-acoustic spectra. Applied Acoustics 60, 505-519.

Nefske, D.J., Wolf, J.A., Jr. and Howell, L.J. (1982). Structural-acoustic finite element analysis of the automobile passenger compartment: a review of current practice. Journal of Sound and Vibration 80, 247-266.

Priede, T. (1971). Origins of automotive vehicle noise. Journal of Sound and Vibration 15, 61-73. 